\section{Perforin-positive Lymphozyten im peripheren Blut bei extrinsischem und intrinsischem Asthma1,2}

V. Arnold, S. Balkow, R. Staats, H. Matthys, W. Luttmann, J. C. Virchow Jr.

Pneumologische Abteilung der Medizinischen Universitätsklinik Freiburg, Deutschland
Zusammenfassung: Asthma bronchiale geht mit einer chronischen, T-Zell-vermittelten bronchialen Entzündung einher. Die Ursache der Erkrankung ist jedoch bis heute unklar. Ähnlich anderen T-Zell-vermittelten chronisch-entzündlichen Erkrankungen, die mit Autoimmunreaktionen assoziiert sind, gibt es auch einzelne Hinweise für Autoimmunphänomene beim Asthma. Da verschiedene Autoimmunerkrankungen mit der Expression von Perforin, einem zytotoxischen Molekül, das von Lymphozyten gebildet wird, einhergehen, sind wir der Hypothese nachgegangen, ob sich beim allergischen und intrinsischen Asthma vermehrt Perforin-produzierende Lymphozyten nachweisen lassen. Dazu wurde intrazellulär die Perforinexpression peripherer Blutlymphozyten von Patienten mit extrinsischem Asthma $(n=13)$, intrinsischen Asthma $(n=7)$ und gesunden Kontrollprobanden $(n=18)$ verglichen. Mittels Durchflusszytometrie wurden die Gesamtlymphozyten identifiziert und mit Hilfe monoklonaler Antikörper in ihre Subpopulationen $\mathrm{CD}^{+}, \mathrm{CD}^{+}$, $\mathrm{CD}^{+}, \mathrm{CD} 16^{+}$und $\mathrm{CD}^{+} 6^{+}$unterteilt. Der Prozentsatz der Perforin-positiven Gesamtlymphozyten war, verglichen mit gesunden Kontrollprobanden, bei Patienten mit allergischem aber auch beim intrinsischem Asthma signifikant erhöht. Verglichen mit gesunden Kontrollprobanden ergab die Untersuchung der Lymphozytensubpopulationen signifikant höhere Prozentsätze an CD3-, CD4-, CD8- und CD56-positiven Zellen beim intrinsischen Asthma, die gleichzeitig auch Perforin exprimierten. Die Perforinexpression in $\mathrm{CD}^{+}$-Zellen beim intrinsischen Asthma war sogar im Vergleich zum allergischen Asthma signifikant erhöht. Daraus ist zu schließen, dass das allergische und das intrinsische Asthma mit einem Anstieg der Perforin-Expression in T-Lymphozyten-Subpopulationen einhergehen.

Increase in Perforin-Positive Peripheral Blood Lymphocytes in Extrinsic and Intrinsic Asthma: The cause of asthma, which has been linked to a chronic, T-cell-mediated bronchial inflammation, remains unclear. A number of other T-lymphocyte-mediated, chronic inflammatory disorders have been associated with autoimmunity and there are data indicating that autoimmune phenomena might also be present in asthma. Expression of perforin, a cytotoxic molecule produced by lymphocytes, has been implicated in the pathogenesis of autoimmune diseases. We therefore tested the hypothesis that allergic and intrinsic asthma might be associated with an increase in lymphocytes producing perforin by comparing the expression

Pneumologie 2000; 54: 468-473

(c) Georg Thieme Verlag Stuttgart · New York

ISSN 0934-8387 of intracellular perforin in peripheral blood lymphocytes of patients with extrinsic asthma $(n=13)$, intrinsic asthma $(n=7)$, and healthy control subjects $(n=18)$. Lymphocytes were identified using flow cytometry and subdivided into CD3(+), CD4(+), CD8(+), CD16(+), and CD56(+) subpopulations after staining with appropriate monoclonal antibodies. The percentage of perforin-positive total lymphocytes was significantly elevated in patients with allergic as well as intrinsic asthma when compared with normal control subjects. Analysis of lymphocyte subpopulations also revealed a significant increase in the percentage of CD3(+), CD4(+), CD8(+), and CD56(+) cells expressing perforin in allergic asthma and a significant increase in the percentage of $\mathrm{CD4}(+)$ and $\mathrm{CD} 56(+)$ cells in intrinsic asthma when compared with healthy control subjects. Perforin expression in CD4(+) cells in intrinsic asthma was also significantly elevated compared with allergic asthma. We conclude that allergic and intrinsic asthma is associated with increased expression of perforin in T-lymphocyte subsets.

\section{Einleitung}

Das Asthma bronchiale ist eine chronisch-entzündliche Erkrankung unbekannter Ursache. Vom klinischen Aspekt her lässt sich Asthma in eine extrinsisch/atopische und eine intrinsisch/nichtallergische Form einteilen. Beim extrinsischen Asthma sind Allergene an der Entstehung der Atemwegsentzündung und des Bronchospasmus beteiligt, wohingegen die Pathogenese des intrinsischen Asthma völlig unklar ist. Sowohl das allergische als auch das intrinsische Asthma gehen mit einer chronischen T-Zell- und Eosinophilen-vermittelten bronchialen Entzündung einher [1,2]. Die Ätiologie dieser Erkrankungen hingegen ist bis heute unklar. Auf klinischen Beobachtungen basierend lässt sich die Hypothese formulieren, dass dem Asthma eine autoimmunologische Komponente zugrunde liegen könnte [3]. Zahlreiche Studien berichten von organ- als auch nichtorganspezifischen Autoantikörpern beim allergischen und/oder intrinsischen Asthma. Zirkulierende Autoantikörper gegen glatte Muskulatur, Schilddrüse, Parietalzellen, Mitochondrien als auch antinukleäre Antikörper und IgG-anti-IgG Antikörper werden in der Literatur beschrieben [4-7]. In einigen dieser Studien waren bei Patienten mit intrinsischem Asthma Autoantikörper öfter

\footnotetext{
${ }^{1}$ Deutsche Fassung von: Increase in Perforin-Positive Peripheral Blood Lymphocytes in Extrinsic and Intrinsic Asthma (Am J Respir Crit Care Med 2000; 161: 182-186)

Zitat nur nach dieser Originalquelle
} 
zu finden $[4,6]$. Zusätzlich war bei Patienten mit Asthma und gleichzeitiger Aspirinintoleranz die Häufigkeit von antinukleären Antikörpern erhöht und diese Patienten zeigten klinische Zeichen einer Autoimmunerkrankung [3]. Andere Studien berichten sowohl beim allergischen als auch beim intrinsischen Asthma von erhöhten Autoantikörperkonzentrationen [4,8]. Andererseits gelang es bisher nicht, diesen Antikörpern im Rahmen der asthmatischen Entzündung eine pathogenetische Bedeutung zuzuordnen.

Bisher gibt es keine Studien, die beim Asthma zellvermittelte Autoimmunphänomene untersuchten. Beim chronisch persistierenden allergischen Asthma, aber insbesondere beim chronisch progredienten intrinsischen Asthma persistieren Entzündungszellen in den Atemwegen. Einige davon besitzen zytolytisches Potenzial. Neben Eosinophilen [9] sind auch aktivierte $\mathrm{CD}^{+}$-T-Lymphozyten [1] und natürliche Killer(NK)-Zellen [10] beim allergischen und/oder intrinsischen Asthma vorhanden. Das Verhältnis von $\mathrm{CD}^{+}-\mathrm{zu}-\mathrm{CD}^{+}$-Zellen im peripheren Blut von Patienten mit intrinsischem Asthma ist im Vergleich zu Patienten mit allergischem Asthma und gesunden Kontrollprobanden erhöht [1]. Auf welche Weise diese Zellen an der Pathogenese des Asthma beteiligt sind, ist bisher allerdings ebenfalls kaum untersucht.

Perforin, ein $60 \mathrm{kD}$ großes, porenformendes Protein, wird von NK-Zellen, gamma-delta $(\gamma \delta)$-Zellen, zytotoxischen CD8+-TLymphozyten und einer kleinen Gruppe von CD4+-T-Lymphozyten gebildet und intrazellulär gespeichert. Bei einer Anzahl chronisch entzündlicher Autoimmunerkrankungen, wie der multiplen Sklerose [17,18], der Takayasu-Arteritis [19] oder autoimmuner Schilddrüsenerkrankungen ließen sich vermehrt perforinhaltige Lymphozyten nachweisen. Perforin kann in zahlreichen Zellen, einschließlich T-Lymphozyten, Apoptose auslösen. Dieses könnte einerseits bei einer entzündlichen Immunantwort dazu beitragen, das entzündliche Infiltrat zu beseitigen [21 - 23], andererseits bei der Elimination von virusinfizierten Zellen eine Rolle spielen [24,25]. Da zahlreiche klinische [26] als auch immunologische [1] Zeichen des intrinsischen Asthma mit einem autoimmunen Geschehen vereinbar sind, prüfen wir nachfolgend die Hypothese, dass beim chronischen Asthma Perforin vermehrt exprimiert wird, was in der Pathogenese der Erkrankungen eine Rolle spielen könnte.

\section{Methoden}

\section{Probanden}

Patienten mit allergischem oder intrinsischem Asthma bronchiale $[1,28]$ wuden für diese Studie ausgewählt. Insgesamt nahmen 13 Probanden mit extrinsischem Asthma (6 Frauen/7 Männer) und einem mittleren Alter von 43 Jahren (22-74 Jahre) an der Studie teil. Die mittlere $\mathrm{FEV}_{1}$ lag bei $77,6+1-$ $5,9 \%(44-105 \%)$. Die sieben intrinsischen Asthmatiker (6 Frauen/1 Mann) waren zwischen 41 und 64 Jahren alt, das mittlere Alter lag bei 55 Jahren. Die mittlere $\mathrm{FEV}_{1}$ lag bei $62,4+/-8,2 \%$ (31-89\%). Für die Kontrollgruppe wurden gesunde Personen (14 Frauen/4 Männer) mit einem durchschnittlichen Alter von 40 Jahren (25-65 Jahre) und einem normalen $\mathrm{FEV}_{1}$ ausgewählt.
Alle Patienten stimmten ihrer Teilnahme an der Studie zu; das Protokoll wurde von der Ethik-Kommission des Universitätsklinikums geprüft und genehmigt.

\section{Zellpräparation mononukleärer Zellen}

Aus $10 \mathrm{ml}$ venösem Blut (von extrinsischen, intrinsischen bzw. gesunden Probanden) versetzt mit 0,2\% EDTA wurden die mononukleären Zellen (MNC) via Ficoll-Dichtegradientenzentrifugation $(1,07 \mathrm{~g} / \mathrm{L})$ isoliert, entsprechend der ausführlichen Beschreibung bei Luttmann u. Mitarb. [28]. Die isolierten MNC wurden zweimal mit PBS/2\% hitzeinaktiviertem FCS gewaschen und anschließend auf eine Konzentration von $1 \times 10^{6}$ Zellen/ml gebracht.

\section{Monoklonale Antikörper}

Spezifische Oberflächenmoleküle wurden mit monoklonalen Antikörpern markiert: anti-humanes CD3-Phycoerythrin (PE) (Klon UCH1; Dako, Hamburg, Deutschland), anti-humanes CD4-PE (Klon EDU-2; Cymbus Biotechnology, Hants, GB), anti-humanes CD8-PE (Klon DK25; Dako), anti-humanes CD16-PE (Klon 3G8; Immunotech, Hamburg, Deutschland) und anti-humanes CD56-PE (Klon B-A19, Diaclone, Besancon, Frankreich). Zur intrazellulären Perforin-Messung wurde der anti-humane Perforin-Fluoresceinisothiocyanat-Antikörper

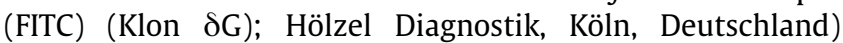
verwendet. Als Isotypkontrollen dienten unspezifische IgGFITC und IgG-PE Antikörper (beide von Dako).

\section{Intrazellulärer Perforinnachweis}

Nach der Markierung der Oberflächenantigene mit anti-CD3, anti-CD4, anti-CD8, anti-CD16 oder anti-CD56 wurden die Zellen in einer 4\%igen Paraformaldehydlösung fixiert, zweimal mit PBS gewaschen und anschließend die Zellmembran mit einer 0,1\%igen Saponinlösung (in PBS) permeabilisiert. Diese Zellen wurden mit FITC-markierten anti-Perforin Antikörpern bei Raumtemperatur $30 \mathrm{~min}$ inkubiert, zweimal gewaschen und zum Schluss durchflusszytometrisch analysiert (FACScan, Becton Dickenson, PC-Lysis II).

\section{Statistische Auswertung}

Die Ergebnisse sind als Mittelwerte \pm Standardfehler (SEM) angegeben. Für die statistische Analyse wurde der MannWhitney-U-Test gewählt. Unterschiede mit $\mathrm{p}<0,05$ wurden als signifikant bezeichnet.

\section{Resultate}

Verteilung der Lymphozyten-Subpopulationen bei extrinsischen und intrinsischen Asthmatikern und gesunden Probanden

Die Verteilung der Lymphozyten-Subpopulationen wurde im Vollblut der verschiedenen Gruppen untersucht. Dazu wurden die Zellen mit fluoreszenzmarkierten Antikörpern gegen CD3, CD4, CD8, CD16 und CD56 inkubiert und durchflusszytometrisch analysiert. Wie in Abb. 1 dargestellt, ergaben sich keine signifikanten Unterschiede in der prozentualen Verteilung der $\mathrm{CD}^{+}-$, $\mathrm{CD}^{+}-$, $\mathrm{CD}^{+}{ }^{-}, \mathrm{CD} 16^{+}-$und $\mathrm{CD}^{2} 6^{+}-$Subpopulation innerhalb der drei untersuchten Gruppen. 


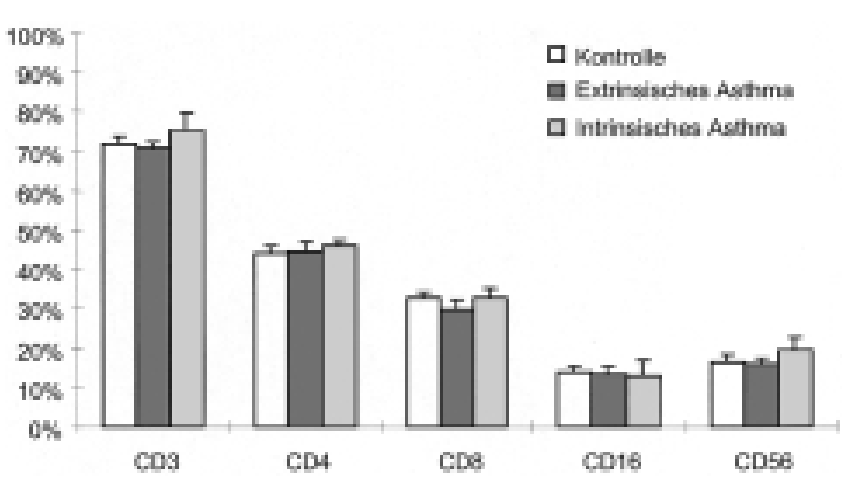

Abb. 1 Zusammensetzung der Lymphozyten des peripheren Bluts. Lymphozyten-Subpopulationen aus dem peripheren Blut von Patienten mit extrinsischem Asthma $(n=13)$, intrinsischem Asthma $(n=7)$ und Normalpersonen $(n=18)$ in \% aller Lymphozyten. Zwischen den einzelnen Gruppen fanden sich keine signifikanten Unterschiede. Die Untersuchungen erfolgten an Vollblut. Die angegebenen Werte entsprechen dem Mittelwert \pm SEM

\section{Perforin-positive Lymphozyten}

Wurden die Zellen aber über Ficoll isoliert, fixiert und permeabilisiert und dann mit dem Anti-Perforin-Antikörper inkubiert, konnte im Vergleich zu den gesunden Probanden $(21,7 \pm 2,5 \%)$ ein signifikant höherer Prozentsatz $(p<0,05)$ an Perforin exprimierenden Zellen bei den extrinsischen $(35,3 \pm 3,5 \%)$ aber auch den intrinsischen Asthmatikern $(37,7 \pm 3,9 \%)$ gemessen werden (Abb.2). Ein signifikanter Unterschied in der Anzahl der Perforin-exprimierenden Zellen bei extrinsischen im Vergleich zu intrinsischen Asthmatikern war aber hingegen nicht zu beobachten.

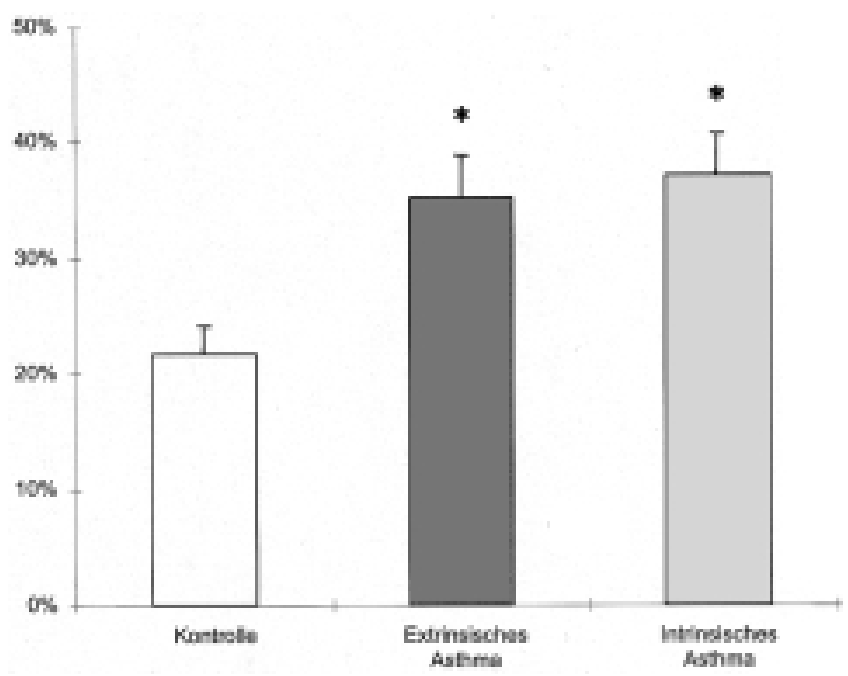

Abb. 2 Prozentsatz der Perforin-positiven Lymphozyten. Vergleich des prozentualen Anteils der Lymphozyten des peripheren Bluts, die intrazellulär Perforin exprimieren. Patienten mit extrinsischem Asthma ( $n=13$ ), intrinsischem Asthma $(n=7)$ und Normalpersonen $(n=18)$. *signifikant gesteigerte Expression von Perforin in Lymphozyten von Patienten mit extrinsischem und intrinsischem Asthma im Vergleich mit Normalpersonen. Die angegebenen Werte entsprechen dem Mittelwert \pm SEM. ${ }^{*} \mathrm{p}<0,05$.

\section{Perforin-Expression in den Lymphozyten-Subpopulationen}

Um die Perforin-exprimierenden Lymphozyten-Subpopulationen weiter zu charakterisieren, wurden die Zellen mit monoklonalen Antikörpern gegen CD3, CD4, CD8, CD16 und CD56 inkubiert, anschließend fixiert und mit Saponin permeabilisiert. Die permeabilisierten Lymphozyten wurden mit dem anti-Perforin-Antikörper inkubiert. Der prozentuale Anteil an Perforin exprimierenden Zellen war in den $\mathrm{CD} 16^{+}-$und CD56+-Zellen am höchsten, aber auch in allen anderen untersuchten Lymphozytenpopulationen wurden Perforin-exprimierende Zellen nachgewiesen, sowohl bei Asthmatikern als auch bei gesunden Probanden. Die Analyse der Daten zeigte keinen Unterschied in der Perforinexpression bei den CD16 Lymphozyten. Es wurde aber eine signifikant erhöhte Perforinexpression bei den $\mathrm{CD}^{+}{ }^{+}, \mathrm{CD}_{4}^{+-}$, $\mathrm{CD}^{+}{ }^{+}$und $\mathrm{CD} 56^{+}$-Lymphozyten bei extrinsischen Asthmatikern im Vergleich zu den intrinsischen Asthmatikern festgestellt. Die gleichen Resultate konnten bei den $\mathrm{CD}^{+}{ }^{-}$und $\mathrm{CD} 56^{+}$-Zellen von intrinsischen Asthmatikern beobachtet werden. Die Population der Perforin exprimierenden $\mathrm{CD}^{+}$- und $\mathrm{CD}^{+}$-Lymphozyten war ebenfalls erhöht, ohne dass diese Ergebnisse statistische Signifikanz aufwiesen. Interessanterweise war der prozentuale Anteil der Perforin- exprimierenden CD4+-Lymphozyten bei intrinsischen Asthmatikern im Vergleich zu den extrinsischen Asthmatikern statistisch signifikant erhöht (Abb. 3).

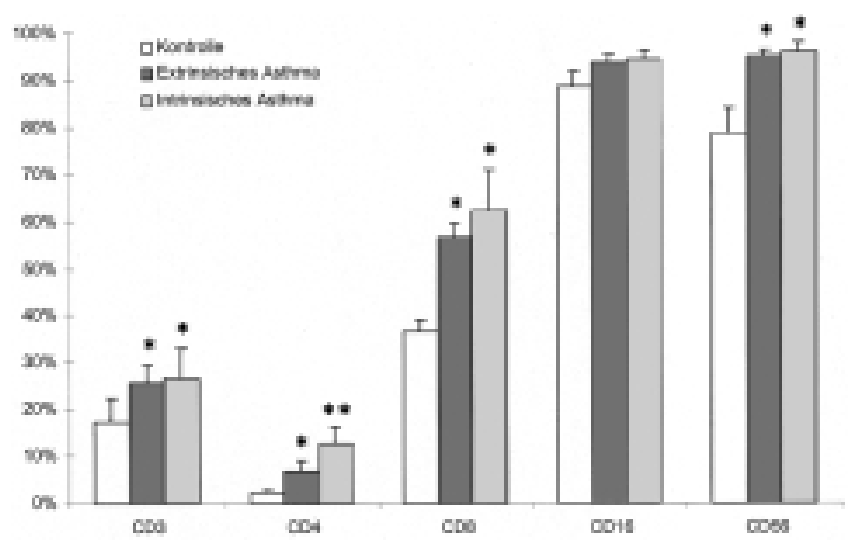

Abb. 3 Prozentsatz der Perforin-positiven Lymphozyten-Subpopulationen. Vergleich des prozentualen Anteils der Lymphozyten-Subpopulationen des peripheren Bluts, die intrazellulär Perforin exprimieren. Sowohl Patienten mit extrinsischem Asthma $(n=13)$ als auch Patienten mit intrinsischem Asthma $(n=7)$ hatten signifikant höhere Anteile Perforin-exprimierender Zellen. Die angegebenen Werte entsprechen dem Mittelwert \pm SEM. ${ }^{*} p<0,05$, Im Gegensatz zu den anderen Lymphozyten-Subpopulationen fand sich bei den CD16 ${ }^{+}$Perforin-positiven Lymphozyten zwischen den untersuchten Gruppen kein signifikanter Unterschied. ${ }^{* *} \mathrm{p}<0,05$ im Vergleich mit Patienten mit allergischem Asthma.

\section{Korrelation mit klinischen Parametern}

Es fanden sich keine Korrelationen zwischen dem Anteil an Perforin-exprimierenden Lymphozyten oder deren Subpopulationen und klinischen Parametern des Asthmas.

Obwohl der prozentuale Anteil Perforin ${ }^{+} / \mathrm{CD}^{+}{ }^{+}$und Perforin ${ }^{+} /$ CD8+-Lymphozyten mit dem Alter der Patienten, die an 
intrinsischem Asthma litten, korreliert waren ( $\mathrm{r}=0,8$ und $r=0,77$ ) war dies nicht mehr statistisch signifikant, wenn die Ergebnisse für multiple Korrelationen korrigiert wurden.

\section{Diskussion}

Die Ätiologie des Asthma bronchiale ist bis heute nicht geklärt. Trotz der Erkenntnis, dass die allergenabhängige Zunahme der bronchialen Entzündung eine wesentliche Rolle bei der Aufrechterhaltung der zellulären Infiltrate der Bronchien beim allergischen Asthma spielen, bleiben zahlreiche Facetten der Erkrankung, insbesondere ihre Neigung zum chronisch progredienten Verlauf unverstanden. Gegenwärtige Hypothesen erklären nicht, weshalb das Asthma auch in Abwesenheit des Allergens persistieren kann und die Mechanismen, die dem intrinsischen Asthma zugrunde liegen, sind weiterhin unklar. Die klinische Beobachtung, dass das Asthma oft einen chronischen, progredienten Verlauf nimmt, dessen Assoziation mit aktivierten T-Lymphozyten beim allergischen als auch beim intrinsischen Asthma [1,2], die zellvermittelte Zerstörung der bronchialen Mukosa und Vorgänge, die als Atemwegs-Remodeling beschrieben werden [29], ferner das positive Ansprechen auf Kortikosteroide, der Nachweis gehäuft vorkommender Autoantikörper [3-7] zusammen mit der gesteigerten Inzidenz des intrinsischen Asthmas bei weiblichen Patienten [30] sowie der unvorhersagbare Verlauf mit Exazerbationen und Remissionen, aber gelegentlich auch rein progredienten Verlaufsformen unterstützen die Hypothese, dass der Pathogenese des Asthma bronchiale auch autoimmunologische Charakteristika zugrunde liegen.

Bislang wurden beim Asthma zellvermittelte autoimmunologische Phänomene nicht untersucht. In der vorliegenden Studie ließ sich zeigen, dass der Prozentsatz Perforin-positiver Lymphozyten im peripheren Blut sowohl beim allergischen als auch beim intrinsischen Asthma verglichen mit normalen Kontrollprobanden gesteigert ist. Der beobachtete Unterschied der Perforinexpression zwischen Asthmatikern und gesunden Kontrollprobanden bleibt auch dann bestehen, wenn $\mathrm{CD}^{+}{ }^{+}, \mathrm{CD}^{+}{ }^{-}, \mathrm{CD}^{+}-$und $\mathrm{CD}^{-} 6^{+}-\mathrm{Lym}$ phozytensubpopulationen getrennt untersucht werden, was als Zeichen dafür verstanden werden kann, dass insbesondere die Perforinexpression dieser Lymphozytensubpopulationen beim Asthma bronchiale hochreguliert ist. Der beobachtete Unterschied der Perforinexpression von Lymphozyten zwischen Patienten mit Asthma und gesunden Kontrollprobanden ist dabei nicht auf eine selektive Umverteilung der Lymphozytensubpopulationen zurückzuführen, da diese zahlenmäßig innerhalb der drei Gruppen identisch waren (Abb.1). So legen unsere Ergebnisse nahe, dass in Anwesenheit ähnlicher Verteilungen von $\mathrm{CD}^{+}{ }^{-}$, $\mathrm{CD}^{+}{ }^{-}, \mathrm{CD}^{+}{ }^{-}, \mathrm{CD}_{16}{ }^{+}$- und $\mathrm{CD} 56^{+}$-Zellen, die vermehrte Perforin-Expression jeder dieser Subpopulationen einen tatsächlichen Anstieg dieser Population im peripheren Blut von Patienten mit Asthma reflektiert.

Kürzlich wurde berichtet, dass der Prozentsatz von $\mathrm{CD}^{+} /$ Perforin $^{+}$-, CD4 ${ }^{+} /$Perforin ${ }^{+}-$und $\mathrm{CD}^{+} /$Perforin $^{+}$-Zellen mit zunehmendem Alter abnimmt [31]. Im Kontrast dazu stehen unsere Beobachtung deutlich erhöhter Perforin-positiver Lymphozyten- und Lymphozytensubpopulationen, vor allem beim intrinsischen Asthma und dies trotz der Tatsache, dass diese Patientengruppe ein höheres Durchschnittsalter gegenüber den normalen Kontrollprobanden aufweist. In Hinblick auf die Erkenntnisse von Rukavina u. Mitarb. [31] dürften die beobachteten Unterschiede der Perforinexpression in unserer Arbeit sogar noch stärker ausfallen, wenn die Werte nach Alter korrigiert würden. Andererseits legt unsere Beobachtung, dass die Perforin-exprimierenden Lymphozytensubpopulationen beim allergischen Asthma und normalen Kontrollprobanden trotz ähnlicher Altersverteilung unterschiedlich sind, nahe, dass die Ergebnisse unserer Untersuchung nicht alleine auf das Alter der beteiligten Probanden zurückgeführt werden können.

Obwohl wir somit erstmals einen erhöhten Prozentsatz an Perforin-positiven Lymphozyten bei Patienten mit Asthma bronchiale zeigen können, gibt unsere Untersuchung keinen Aufschluss über die funktionelle oder klinische Bedeutung dieser Zellen bei den untersuchten Erkrankungen. Inwieweit diese Zellen tatsächlich zytotoxisches Potenzial besitzen bleibt unklar und es gibt bislang keine funktionellen Studien beim Menschen, die zur Beantwortung dieser Frage herangezogen werden könnten.

Gesunde Freiwillige exprimieren Perforin intrazellulär in NKund $\mathrm{CD}^{+}$-Zellen, aber nur zu einem geringen Prozentsatz in CD4+-Lymphozyten [32]. Nakata u. Mitarb. konnten mittels Immunzytochemie in unstimulierten $\mathrm{CD}^{+}$-T-Lymphozyten gesunder Kontrollpersonen überhaupt keine Expression von Perforin nachweisen [16]. Dies steht im Gegensatz zu unseren Ergebnissen, die einen geringen Prozentsatz an Perforinpositiven $\mathrm{CD}^{+}$-Zellen in gesunden Kontrollpersonen zeigen. Ein erhöhter Anteil Perforin ${ }^{+} / \mathrm{CD}^{+}-$Zellen wurde bei der infektiösen Mononukleose [16] und nach Therapie beim Morbus Hodgkin [33] beschrieben. Auch bei der Wegnerschen Granulomatose zeigen periphere Lymphozyten einen vermehrten Anteil von Perforin ${ }^{+} / \mathrm{CD}^{+}{ }^{+}$-Zellen (M. Schlesier, Freiburg, persönliche Mitteilung). Interessanterweise konnte in unserer Studie der höchste Anteil dieser Zellen bei Patienten mit intrinsischem Asthma gefunden werden. Da bislang nur wenige Informationen über die physiologische Rolle und vermeintliche zytotoxische Eigenschaften CD4+ MHC II-Klasse restringierter T-Zellen bekannt ist, lässt sich über die Rolle Perforin $^{+}-/ \mathrm{CD}^{+}{ }^{+}$-Zellen beim Asthma bronchiale nur spekulieren. Für mehrere chronisch entzündliche Erkrankungen aus dem Formenkreis der Autoimmunerkrankungen wurde eine erhöhte Perforinexpression beschrieben. Hierzu zählen die multiple Sklerose [17,18], die Takayasu-Arteriitis [19], Autoimmunerkrankungen der Schilddrüse [20] und der Morbus Crohn. Perforin wurde in diesen Fällen auf mononukleären Zellen, CD4+-, CD8 ${ }^{+}$, , CD16 ${ }^{+},, \gamma / \delta$-T-Zellen oder NK-Zellen nachgewiesen. Unsere Untersuchungen an Patienten mit Asthma bronchiale ergaben erhöhte Werte der potenziell zytotoxischen $\mathrm{CD}^{+}$- und $\mathrm{CD}^{+}$-T-Lymphozyten im peripheren Blut. Dieses Ergebnis bestätigt unsere Hypothese, dass auch beim Asthma bronchiale eine Autoimmunreaktion an der Pathogenese beteiligt sein dürfte.

Es gibt verschiedene Möglichkeiten, durch die der gesteigerte Anteil Perforin-positiver T-Lymphozyten mit der chronischen Entzündung beim Asthma verknüpft sein könnte. Die systemische Gabe von Interleukin-2 (IL-2) im Rahmen einer

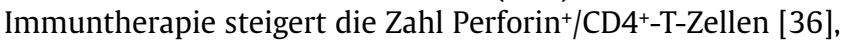
was darauf hinweist, dass der Effekt der IL-2-Immuntherapie über zytolytische Lymphozyten vermittelt wird. Vergleichbar dazu wurde Perforin-vermittelte Zytotoxizität mit dem durch 
IL-2 induzierten „vascular leak syndrome“ in Verbindung gebracht, bei dem IL-2 offenbar die Perforin-Expression steigert [37]. Erhöhte IL-2-Konzentrationen wurden sowohl in der BAL beim intrinsischen Asthma als auch nach segmentaler Allergenprovokation beim allergischen Asthma nachgewiesen [2]. Folglich könnten erhöhte IL-2-Konzentrationen, wie beim Asthma in anderen Studien nachgewiesen, zur Zahl der Perforin-positiven Lymphozyten in unserer Studienpopulation beigetragen haben.

Andererseits ist aus Tiermodellen des Lupus erythematodes bekannt, dass Perforin-defiziente Tiere einen schwereren Krankheitsverlauf nehmen, was darauf hinweist, dass der zytolytischen lymphoiden Regulation eine zentrale Bedeutung bei der Immun-Homöostase zukommt [38]. Inwieweit der erhöhte Anteil Perforin ${ }^{+}$-Lymphozyten unserer Patienten Zeichen einer gesteigerten zytolytischen Aktivität im Rahmen der Asthmapathogenese sind oder stattdessen eine Reaktion des Immunsystems zur Elimination reichlich vorhandener Entzündungszellen darstellt, lässt sich derzeit nicht zweifelsfrei beantworten.

Zusammenfassend zeigen unsere Ergebnisse, dass das Asthma bronchiale mit einem gesteigerten Anstieg potenziell zytolytischer Perforin ${ }^{+}$-Lymphozyten vergesellschaftet ist. Die funktionelle Relevanz dieser Befunde in der Pathogenese des Asthmas ist unklar. Auch wenn eine Verbindung dieser Zellen mit der Pathologie beim Asthma aus unseren Ergebnissen möglich scheint, so ist ein kausaler Zusammenhang zwischen Asthma und Perforinexpression offen und sollte weiter untersucht werden.

\section{Literatur}

${ }^{1}$ Walker C, Bode E, Boer L, Hansel TT, Blaser K, Virchow Jr JC. Allergic and nonallergic asthmatics have distinct patterns of Tcell activation and cytokine production in peripheral blood and bronchoalveolar lavage. Am Rev Respir Dis 1992; 146: 109-115

${ }^{2}$ Virchow Jr JC, Walker C, Hafner D, Kortsik C, Werner P, Matthys $\mathrm{H}$, Kroegel C. T cells and cytokines in bronchoalveolar lavage fluid after segmental allergen provocation in atopic asthma. Am J Respir Crit Care Med 1995; 151: 960-968

${ }^{3}$ Szczeklik A, Nizankowska E, Serafin A, Dyczek A, Duplaga M, Musial J. Autoimmune phenomena in bronchial asthma with special reference to aspirin intolerance. Am J Respir Crit Care Med 1995; 152: $1753-1756$

${ }^{4}$ Turner-Warwick M, Haslam P. Smooth muscle antibody in bronchial asthma. Clin Exp Immunol 1970; 7: 31 - 38

${ }^{5}$ Egeskjold EM, Permin H, Nielsen I, Sorensen HJ, Osterballe O, Kallerup HE. Anti-IgG antibodies and antinuclear antibodies in allergic patients. European Journal of Allergy \& Clinical Immunology 1981; 36: $573-581$

${ }^{6}$ Lidor Y, Topilsky M, Spitzer SA, Yehoshua H. Autoimmune antibodies in intrinsic (non-atopic) asthma. Annals of Allergy 1980; 44: 296-298

${ }^{7}$ Menon P, Menon V, Hilman BC, Wolf R, Bairnsfather L. Antinuclear antibodies and anticytoplasmic antibodies in bronchial asthma. J Allergy Clin Immunol 1989; 84: 937-943

${ }^{8}$ Venter JC, Fraser CM, Harrison LC. Autoantibodies to beta 2adrenergic receptors: a possible cause of adrenergic hyporesponsiveness in allergic rhinitis and asthma. Science 1980; 207: $1361-1363$

${ }^{9}$ Bousquet J, Chanez P, Lacoste JY, Barneon G, Ghavanian N, Enander I, Venge P, Ahlstedt S, Simony-Lafontaine J, Godard P et al. Eosinophilic inflammation in asthma. New England Journal of Medicine 1990; 323: 1033-1039

${ }^{10}$ Krejsek J, Kral B, Vokurkova D, Derner V, Touskova M, Parakova Z, Kopecky O. Decreased peripheral blood gamma delta T cells in patients with bronchial asthma. European Journal of Allergy \& Clinical Immunology 1998; 53: $73-77$

${ }^{11}$ Lichtenheld MG, Olsen KJ, Lu P, Lowrey DM, Hameed A, Hengartner H, Podack ER. Structure and function of human perforin. Nature 1988; 335: 448-451

12 Millard PJ, Henkart MP, Reynolds CW, Henkart PA. Purification and properties of cytoplasmic granules from cytotoxic rat LGL tumors. J Immunol 1984; 132: 3197 - 3204

${ }^{13}$ Shinkai Y, Takio K, Okumura K. Homology of perforin to the ninth component of complement (C9). Nature 1988; 334: 525527

${ }^{14}$ Koizumi H, Liu CC, Zheng LM, Joag SV, Bayne NK, Holoshitz J, Young JD. Expression of perforin and serine esterases by human gamma/delta T cells. J Exp Med 1991; 173: 499-502

${ }^{15}$ Konjevic G, Schlesinger B, Cheng L, Olsen KJ, Podack ER, Spuzic I. Analysis of perforin expression in human peripheral blood lymphocytes, CD56+ natural killer cell subsets and its induction by interleukin-2. Immunol Invest 1995; 24: 499-507

${ }^{16}$ Nakata M, Kawasaki A, Azuma M, Tsuji K, Matsuda H, Shinkai Y, Yagita H, Okumura K. Expression of perforin and cytolytic potential of human peripheral blood lymphocyte subpopulations. Int Immunol 1992; 4: 1049-1054

${ }^{17}$ Rubesa G, Podack ER, Sepcic J, Rukavina D. Increased perforin expression in multiple sclerosis patients during exacerbation of disease in peripheral blood lymphocytes. J Neuroimmunol 1997; 74: $198-204$

${ }^{18}$ Matusevicius D, Kivisakk P, Navikas V, Soderstrom M, Fredrikson $\mathrm{S}$, Link $\mathrm{H}$. Interleukin-12 and perforin mRNA expression is augmented in blood mononuclear cells in multiple sclerosis. Scand J Immunol 1998; 47: 582 - 590

${ }^{19}$ Seko Y, Minota S, Kawasaki A, Shinkai Y, Maeda K, Yagita H, Okumura K, Sato O, Takagi A, Tada Y et al. Perforin-secreting killer cell infiltration and expression of a 65-kD heat-shock protein in aortic tissue of patients with Takayasu's arteritis. J Clin Invest 1994; 93: 750-758

${ }^{20} \mathrm{Wu}$ Z, Podack ER, McKenzie JM, Olsen KJ, Zakarija M. Perforin expression by thyroid-infiltrating $\mathrm{T}$ cells in autoimmune thyroid disease. Clin Exp Immunol 1994; 98: 470-477

${ }^{21}$ Darmon AJ, Nicholson DW, Bleackley RC. Activation of the apoptotic protease CPP32 by cytotoxic T-cell-derived granzyme B. Nature 1995; 377: $446-448$

22 Darmon AJ, Ley TJ, Nicholson DW, Bleackley RC. Cleavage of CPP32 by granzyme $B$ represents a critical role for granzyme $B$ in the induction of target cell DNA fragmentation. J Biol Chem 1996; 271: 21709-21712

${ }^{23}$ Spaner D, Raju K, Radvanyi L, Lin Y, Miller RG. A role for perforin in activation-induced cell death. J Immunol 1998; 160: 26552664

${ }^{24}$ Topham DJ, Tripp RA, Doherty PC. CD8+ T cells clear influenza virus by perforin of Fas-dependent processes. J Immunol 1997; 159: $5197-5200$

${ }^{25}$ Sambhara S, Switzer I, Kurichh A, Miranda R, Urbanczyk L, James O, Underdown B, Klein M, Burt D. Enhanced antibody and cytokine responses to influenza viral antigens in perforindeficient mice. Cellular Immunology 1998; 187: 13-18

${ }^{26}$ Virchow Jr JC, Kroegel C, Walker C, Matthys H. Inflammatory determinants of asthma severity: mediator and cellular changes in bronchoalveolar lavage fluid of patients with severe asthma. J Allergy Clin Immunol 1996; 98: S27 - 33

${ }^{27}$ Anonymous. International consensus report on diagnosis and treatment of asthma. European Respiratory Journal 1992; 5: $601-641$ 
${ }^{28}$ Luttmann W, Herzog V, Virchow Jr JC, Matthys H, Thierauch KH, Kroegel C. Prostacyclin modulates granulocyte/macrophage colony-stimulating factor release by human blood mononuclear cells. Pulm Pharmacol 1996; 9: 43-48

${ }^{29}$ Leung DY. Atopic dermatitis: the skin as a window into the pathogenesis of chronic allergic diseases. J Allergy Clin Immunol 1995; 96: $302-318$

${ }^{30}$ Virchow C. Intrinsic asthma; symptoms, IgE serum levels, pathophysiology. Prax Pneumol 1973; 27: 578-591

${ }^{31}$ Rukavina D, Laskarin G, Rubesa G, Strbo N, Bedenicki I, Manestar D, Glavas M, Christmas SE, Podack ER. Age-related decline of perforin expression in human cytotoxic $\mathrm{T}$ lymphocytes and natural killer cells. Blood 1998; 92: 2410-2420

32 Rutella S, Rumi C, Lucia MB, Etuk B, Cauda R, Leone G. Flow cytometric detection of perforin in normal human lymphocyte subpopulations defined by expression of activation/differentiation antigens. Immunology Letters 1998; 60: 51 - 55

${ }^{33}$ Watanabe N, De Rosa CC, Cmelak A, Hoppe R, Herzenberg LA, Roederer M. Long-term depletion of naive $T$ cells in patients treated for Hodgkin's disease. Blood 1997; 90: 3662-3672

${ }^{34}$ Vergelli M, Hemmer B, Muraro PA, Tranquill L, Biddison WE, Sarin A, McFarland HF, Martin R. Human autoreactive CD4+ T cell clones use perforin - or Fas/Fas ligand-mediated pathways for target cell lysis. J Immunol 1997; 158: 2756 - 2761

${ }^{35}$ Muller S, Lory J, Corazza N, Griffiths GM, Z'graggen K, Mazzucchelli L, Kappeler A, Mueller C. Activated CD4+ and CD8+ cytotoxic cells are present in increased numbers in the intestinal mucosa from patients with active inflammatory bowel disease. Am J Pathol 1998; 152: 261 - 268

${ }^{36}$ Truica CI, Hansen CH, Garvin DF, Meehan KR. Idiopathic giant cell myocarditis after autologous hematopoietic stem cell transplantation and interleukin-2 immunotherapy: a case report. Cancer 1998; 83: 1231 - 1236

${ }^{37}$ Rafi AQ, Zeytun A, Bradley MJ, Sponenberg DP, Grayson RL, Nagarkatti M, Nagarkatti PS. Evidence for the involvement of Fas ligand and perforin in the induction of vascular leak syndrome. J Immunol 1998; 161: $3077-3086$

38 Peng SL, Moslehi J, Robert ME, Craft J. Perforin protects against autoimmunity in lupus-prone mice. J Immunol 1998; 160: 652 660

\section{PD Dr. J. C. Virchow}

Abteilung Pneumologie

Medizinische Universitätsklinik

Hugstetter Str. 55

79106 Freiburg

E-mail: virchow@med1.ukl.uni-freiburg.de 